\title{
NOISE ENHANCED DETECTION IN THE RESTRICTED BAYESIAN FRAMEWORK
}

\author{
Suat Bayram*, Sinan Gezici*, H. Vincent Poor ${ }^{\dagger}$ \\ * Dept. of Electrical and Electronics Engineering, Bilkent University, Bilkent, Ankara 06800, Turkey \\ $\dagger$ Department of Electrical Engineering, Princeton University, Princeton, New Jersey 08544, USA
}

\begin{abstract}
Effects of additive independent noise are investigated for suboptimal detectors according to the restricted Bayes criterion. The statistics of optimal additive noise are characterized. Also, sufficient conditions for improvability or nonimprovability of detection via additive noise are obtained. A detection example is presented to study the theoretical results.
\end{abstract}

Index Terms - Detection, restricted Bayes, minimax, noise enhanced detection, stochastic resonance.

\section{INTRODUCTION}

Performance of some suboptimal detectors can be enhanced by adding independent noise to their observations. Improving the performance of a detector by adding a stochastic signal to its observation can be considered in the framework of stochastic resonance $(\mathrm{SR})$, which can be regarded as noise benefits related to signal transmission in nonlinear systems (please refer to [1]-[3] and references therein for more details). In other words, for certain detectors, addition of controlled "noise" can improve detection performance. Such noise benefits can be in various forms, such as an increase in output signal-tonoise ratio (SNR) [4], a decrease in probability of error [5], or an increase in probability of detection under a false-alarm rate constraint [3], [6], [7].

The effects of additive noise on detection performance are studied in [3] and [6] in the Neyman-Pearson framework, and it is shown that the optimal additive noise can be represented by a randomization of at most two different signal values. In [5], noise enhanced detection is investigated according to the Bayesian criterion under uniform cost assignment. It is shown that the optimal noise that minimizes the probability of decision error has a constant value, and a Gaussian mixture example is presented to illustrate the improvability of a suboptimal detector via adding constant noise. Also, the studies in [8] and [9] consider the minimax criterion and investigate the effects of additive noise on suboptimal detectors.

Although both the Bayesian and the minimax frameworks have been considered for the noise enhanced detection problem, no studies have considered the restricted Bayes criterion [10]. Under the Bayesian criterion, the prior information is precisely known, whereas it is not available under the minimax criterion. However, having prior information with some uncertainty is the most common situation, and the restricted Bayes criterion is well-suited to that case [10], [11]. In the restricted Bayesian framework, the aim is to minimize the

This work was supported in part by the U.S. Office of Naval Research under Grant N00014-09-1-0342.
Bayes risk under a constraint on the individual conditional risks [10]. Depending on the value of the constraint, the restricted Bayes criterion covers the Bayesian and minimax criteria as special cases [11].

In this study, noise enhanced detection is studied in the restricted Bayesian framework. First, a generic problem formulation is presented (Section 2). Then, the statistics of optimal additive noise are obtained, and various sufficient conditions are derived to specify when the performance of a detector can or cannot be enhanced via additive noise in the restricted Bayesian framework (Section 3). Finally, a detection example is presented to illustrate the theoretical results, and concluding remarks are made (Section 4).

\section{PROBLEM FORMULATION AND MOTIVATION}

Consider the following $M$-ary hypothesis-testing problem:

$$
\mathcal{H}_{i}: p_{i}^{\boldsymbol{X}}(\boldsymbol{x}), \quad i=0,1, \ldots, M-1,
$$

where $p_{i}^{\boldsymbol{X}}(\boldsymbol{x})$ denotes the probability density function (PDF) of the observation under hypothesis $\mathcal{H}_{i}$ and the observation $\boldsymbol{x}$ is a vector with $K$ components; that is, $\boldsymbol{x} \in \mathbb{R}^{K}$. The prior probabilities of the hypotheses are denoted by $\pi_{0}, \pi_{1}, \ldots, \pi_{M-1}$. Also, a generic decision rule is defined as

$$
\phi(\boldsymbol{x})=i, \text { if } \boldsymbol{x} \in \Gamma_{i},
$$

for $i=0,1, \ldots, M-1$, where $\Gamma_{0}, \Gamma_{1}, \ldots, \Gamma_{M-1}$ form a partition of the observation space $\Gamma$.

In some cases, addition of noise to observations can improve the performance of a suboptimal decision rule (detector) [3], [6]. By adding noise $\boldsymbol{n}$ to the original data $\boldsymbol{x}$, the modified observation is formed as $\boldsymbol{y}=\boldsymbol{x}+\boldsymbol{n}$, where $\boldsymbol{n}$ has a PDF denoted by $p_{\boldsymbol{N}}(\cdot)$, and is independent of $\boldsymbol{x}$. As in [3] and [6], it is assumed that the detector in (2) is fixed, and that the only means for improving the performance of the detector is to optimize the additive noise $\boldsymbol{n}$. In other words, the aim is to find the best $p_{N}(\cdot)$ according to the restricted Bayes criterion [10]; namely, to minimize the Bayes risk under certain constraints on the conditional risks, as specified below.

$$
\begin{aligned}
& \min _{p_{\boldsymbol{N}}(\cdot)} \sum_{i=0}^{M-1} \pi_{i} \mathrm{R}_{i}^{\boldsymbol{y}}(\phi) \\
& \text { subject to } \max _{i \in\{0,1, \ldots, M-1\}} \mathrm{R}_{i}^{\boldsymbol{y}}(\phi) \leq \alpha
\end{aligned}
$$

where $\alpha$ represents the upper limit on the conditional risks, $\sum_{i=0}^{M-1} \pi_{i} \mathrm{R}_{i}^{\boldsymbol{y}}(\phi) \triangleq r^{\boldsymbol{y}}(\phi)$ is the Bayes risk and $\mathrm{R}_{i}^{\boldsymbol{y}}(\phi)$ represents the conditional risk of $\phi$ given $\mathcal{H}_{i}$ for the noise modified 
observation $\boldsymbol{y}$. More specifically, $\mathrm{R}_{i}^{\boldsymbol{y}}(\phi)=\sum_{j=0}^{M-1} C_{j i} \mathrm{P}_{i}^{\boldsymbol{y}}\left(\Gamma_{j}\right)$, where $\mathrm{P}_{i}^{\boldsymbol{y}}\left(\Gamma_{j}\right)$ denotes the probability that $\boldsymbol{y} \in \Gamma_{j}$ when $\mathcal{H}_{i}$ is the true hypothesis, and $C_{j i}$ is the cost of deciding $\mathcal{H}_{j}$ when $\mathcal{H}_{i}$ is true. In the restricted Bayes formulation, any undesired effects due to the uncertainty of prior probabilities can be eliminated via parameter $\alpha$, which can also be considered as an upper bound for the Bayes risk [11].

Two main motivations for studying the effects of additive noise on detector performance are as follows. First, the optimal detectors according to the restricted Bayes criterion are difficult to obtain, or require intense computations [11]. Therefore, a suboptimal detector with additive noise can provide reasonable performance with low complexity in some cases. Second, it is of theoretical interest to investigate the improvements that can be achieved via additive noise.

\section{OPTIMAL ADDITIVE NOISE AND (NON)IMPROVABILITY CONDITIONS}

In order to obtain the optimal additive noise from (3), an alternative expression for $\mathrm{R}_{i}^{\boldsymbol{y}}(\phi)$ can be obtained first. Since the additive noise $\boldsymbol{n}$ is independent of the observation $\boldsymbol{x}, \mathrm{P}_{i}^{\boldsymbol{y}}\left(\Gamma_{j}\right)$ is given by $\int_{\Gamma_{j}} \int_{\mathbb{R}^{K}} p_{\boldsymbol{N}}(\boldsymbol{n}) p_{i}^{\boldsymbol{X}}(\boldsymbol{y}-\boldsymbol{n}) d \boldsymbol{n} d \boldsymbol{y}$. Then, $\mathrm{R}_{i}^{\boldsymbol{y}}(\phi)=$ $\sum_{j=0}^{M-1} C_{j i} \mathrm{P}_{i}^{\boldsymbol{y}}\left(\Gamma_{j}\right)$ can be expressed as

$$
\mathrm{R}_{i}^{\boldsymbol{y}}(\phi)=\sum_{j=0}^{M-1} C_{j i} \mathrm{E}\left\{F_{i j}(\boldsymbol{N})\right\}=\mathrm{E}\left\{F_{i}(\boldsymbol{N})\right\},
$$

where $N$ is the random variable representing the additive noise, $F_{i j}(\boldsymbol{N}) \triangleq \int_{\Gamma_{j}} p_{i}^{\boldsymbol{X}}(\boldsymbol{y}-\boldsymbol{N}) d \boldsymbol{y}$ and $F_{i}(\boldsymbol{N}) \triangleq$ $\sum_{j=0}^{M-1} C_{j i} F_{i j}(\boldsymbol{N})$. Thus, (3) can be reformulated as

$$
\begin{aligned}
& \min _{p_{\boldsymbol{N}}(\cdot)} \sum_{i=0}^{M-1} \pi_{i} \mathrm{E}\left\{F_{i}(\boldsymbol{N})\right\} \triangleq \mathrm{E}\{F(\boldsymbol{N})\} \\
& \text { subject to } \max _{i \in\{0,1, \ldots, M-1\}} \mathrm{E}\left\{F_{i}(\boldsymbol{N})\right\} \leq \alpha
\end{aligned}
$$

where $F(\boldsymbol{N}) \triangleq \sum_{i=0}^{M-1} \pi_{i} F_{i}(\boldsymbol{N})$. Note that under uniform cost assignment (UCA); that is, when $C_{j i}=1$ for $j \neq i$, and $C_{j i}=0$ for $j=i, F_{i}(\boldsymbol{N})$ becomes equal to $1-F_{i i}(\boldsymbol{N})$.

It is noted from (4) that, in the absence of additive noise $\boldsymbol{n}$, the original conditional risks are given by $\mathrm{R}_{i}^{\boldsymbol{x}}(\phi)=F_{i}(\mathbf{0})$ for $i=0,1, \ldots, M-1$. Similarly, the original Bayes risk is defined as $r^{\boldsymbol{x}}(\phi) \triangleq F(\mathbf{0})$ in the absence of noise (cf. (5)).

The optimization problem in (5) seems quite difficult to solve since it requires a search over all possible noise PDFs. However, it is shown in the following that an optimal additive noise PDF can be represented by a discrete probability distribution with at most $M$ mass points in most practical cases. To that aim, it is first assumed that the possible values that the additive noise can take satisfy $\boldsymbol{a} \preceq \boldsymbol{n} \preceq \boldsymbol{b}$ for certain $\boldsymbol{a}$ and $\boldsymbol{b}$ values; that is, $n_{j} \in\left[a_{j}, b_{j}\right]$ for $j=1, \ldots, K$, which is a reasonable assumption as the additive noise cannot take infinitely large positive/negative values. Then, the following proposition states the discrete nature of the optimal additive noise, which can be proven similarly to a result in [6].
Proposition 1: If $F_{i}(\cdot)$ in (5) are continuous functions, then the optimal additive noise PDF can be expressed as

$$
p_{N}(\boldsymbol{n})=\sum_{l=1}^{M} \lambda_{l} \delta\left(\boldsymbol{n}-\boldsymbol{n}_{l}\right)
$$

where $\sum_{l=1}^{M} \lambda_{l}=1$ and $\lambda_{l} \geq 0$ for $l=1,2, \ldots, M$.

From Proposition 1, the optimization problem in (5) can be simplified as

$$
\begin{aligned}
& \min _{\left\{\lambda_{l}, \boldsymbol{n}_{l}\right\}_{l=1}^{M}} \sum_{l=1}^{M} \lambda_{l} F\left(\boldsymbol{n}_{l}\right) \\
& \text { subject to } \max _{i \in\{0,1, \ldots, M-1\}} \sum_{l=1}^{M} \lambda_{l} F_{i}\left(\boldsymbol{n}_{l}\right) \leq \alpha \\
& \sum_{l=1}^{M} \lambda_{l}=1, \quad \lambda_{l} \geq 0, \quad l=1,2, \ldots, M .
\end{aligned}
$$

The optimization in (7) is over a set of variables instead of functions (cf. (5)). However, it can still be a nonconvex optimization problem in general; hence, global optimization techniques, such as particle-swarm optimization (PSO) and differential evolution can be applied [12]. In Section 4, PSO is employed to obtain the PDF of optimal additive noise.

Next, sufficient conditions are derived to determine when it is (not) possible to improve the performance of a detector via additive independent noise. In that respect, a detector is called improvable if there exists a noise PDF that satisfies $\mathrm{E}\{F(\boldsymbol{N})\}<F(\mathbf{0})$ and $\max \mathrm{E}\left\{F_{i}(\boldsymbol{N})\right\} \leq \alpha$; otherwise, it is called nonimprovable. First, sufficient conditions for nonimprovability are obtained.

Proposition 2: Assume that there exists $i \in\{0,1, \ldots, M-$ 1\} for which $F_{i}(\boldsymbol{n}) \leq \alpha$ implies $F(\boldsymbol{n}) \geq F(\mathbf{0})$ for all $\boldsymbol{n} \in \mathcal{S}_{\mathrm{n}}$, where $\mathcal{S}_{\mathrm{n}}$ is a convex set ${ }^{1}$ consisting of all possible values of additive noise $\boldsymbol{n}$. If $F_{i}(\boldsymbol{n})$ and $F(\boldsymbol{n})$ are convex over $\mathcal{S}_{\mathrm{n}}$, then the detector is nonimprovable.

Proof: It relies on the applications of Jensen's inequality.

The importance of Proposition 2 lies in the fact that whenever the conditions in the proposition are satisfied, no additive noise can improve the detector performance; hence, unnecessary efforts in trying to solve (7) can be prevented.

In order to derive sufficient conditions for improvability, it is assumed that $F(\boldsymbol{x})$ and $F_{i}(\boldsymbol{x})$ for $i=0,1, \ldots, M-1$ are second-order continuously differentiable around $\boldsymbol{x}=\mathbf{0}$. In addition, define $f_{j}^{(1)}(\boldsymbol{x}, \mathbf{z}) \triangleq \sum_{i=1}^{K} z_{i} \frac{\partial F_{j}(\boldsymbol{x})}{\partial x_{i}}, f^{(1)}(\boldsymbol{x}, \mathbf{z}) \triangleq$ $\sum_{i=1}^{K} z_{i} \frac{\partial F(\boldsymbol{x})}{\partial x_{i}}, f_{j}^{(2)}(\boldsymbol{x}, \mathbf{z}) \triangleq \sum_{l=1}^{K} \sum_{i=1}^{K} z_{l} z_{i} \frac{\partial^{2} F_{j}(\boldsymbol{x})}{\partial x_{l} \partial x_{i}}$, and $f^{(2)}(\boldsymbol{x}, \mathbf{z}) \triangleq \sum_{l=1}^{K} \sum_{i=1}^{K} z_{l} z_{i} \frac{\partial^{2} F(\boldsymbol{x})}{\partial x_{l} \partial x_{i}}$ where $x_{i}$ and $z_{i}$ represent the $i$ th components of $\boldsymbol{x}$ and $\mathbf{z}$, respectively. Then, the following proposition provides improvability conditions based on the first and second order derivatives.

Proposition 3: Suppose $F_{k}(\mathbf{0})=\alpha$ and $F_{i}(\mathbf{0})<\alpha$ for $i=0,1, \ldots, k-1, k+1, \ldots, M-1$. Then, the detector is improvable

${ }^{1}$ Since the convex combination of individual noise components are obtained via randomization [13], $\mathcal{S}_{\mathrm{n}}$ can be modeled as convex. 
- if there exists a $K$-dimensional vector $\mathrm{z}$ such that $f_{k}^{(1)}(\boldsymbol{x}, \mathbf{z}) f^{(1)}(\boldsymbol{x}, \mathbf{z})>0$ is satisfied at $\boldsymbol{x}=\mathbf{0}$; or,

- if there exists a $K$-dimensional vector $\mathbf{z}$ such that $f^{(1)}(\boldsymbol{x}, \mathbf{z})>0, f_{k}^{(1)}(\boldsymbol{x}, \mathbf{z})<0$ and $f^{(2)}(\boldsymbol{x}, \mathbf{z}) f_{k}^{(1)}(\boldsymbol{x}, \mathbf{z})$ $>f_{k}^{(2)}(\boldsymbol{x}, \mathbf{z}) f^{(1)}(\boldsymbol{x}, \mathbf{z})$ are satisfied at $\boldsymbol{x}=\mathbf{0} .^{2}$

Whenever any of the results in Proposition 3 holds, the detector is improvable. Therefore, the optimization problem in (7) can be solved to specify the optimal additive noise. Although Proposition 3 considers that the maximum of the original conditional risks, $F_{0}(\mathbf{0}), F_{1}(\mathbf{0}), \ldots, F_{M-1}(\mathbf{0})$, is unique and equal to $\alpha$, the results in Proposition 3 can be extended to cover other cases as well, which is not pursued here due to space limitations.

\section{NUMERICAL RESULTS AND CONCLUSIONS}

In this section, a binary hypothesis-testing problem is studied in order to provide an example of the results presented in the previous section. The hypotheses $\mathcal{H}_{0}$ and $\mathcal{H}_{1}$ are defined as

$$
\mathcal{H}_{0}: x=v, \quad \mathcal{H}_{1}: x=A+v,
$$

where $x \in \mathbb{R}$ and $A>0$ is a known scalar value. In addition, $v$ is Gaussian mixture noise with the PDF $p_{V}(x)=$ $\sum_{i=1}^{M} w_{i} \psi_{i}\left(x-\mu_{i}\right)$, where $w_{i} \geq 0$ for $i=1, \ldots, M$, $\sum_{i=1}^{M} w_{i}=1$, and $\psi_{i}(x)=\frac{1}{\sqrt{2 \pi} \sigma_{i}} \exp \left(\frac{-x^{2}}{2 \sigma_{i}^{2}}\right)$ for $i=$ $1, \ldots, M$. In addition, the detector is described by

$$
\phi(y)=\left\{\begin{array}{ll}
1, & y \geq A / 2 \\
0, & y<A / 2
\end{array},\right.
$$

where $y=x+n$, with $n$ representing the additive independent noise term.

Based on the definitions in Section 3, $F_{0}(\boldsymbol{x})$ and $F_{1}(\boldsymbol{x})$ can be obtained as

$$
F_{k}(x)=1-\sum_{i=1}^{M} w_{i} Q\left(\frac{-A / 2+a_{k}\left(x+\mu_{i}\right)}{\sigma_{i}}\right),
$$

for $k=0,1$, where $a_{0}=1, a_{1}=-1$, and $Q(x)=$ $\frac{1}{\sqrt{2 \pi}} \int_{x}^{\infty} \mathrm{e}^{-t^{2} / 2} d t$ denotes the $Q$-function.

In practice, the example described above can be encountered in detection of communications signals in the presence of co-channel interference, which can result in Gaussian mixture noise at the receiver [14].

In the simulations, UCA is employed and two cases are considered for the prior probabilities: i) $\pi_{0}=0.9, \pi_{1}=0.1$ (unequal priors), ii) $\pi_{0}=\pi_{1}=0.5$ (equal priors). Also, symmetric Gaussian mixture noise with $M=4$ is considered for noise $v$ in (8), where the mean values of the Gaussian components in the mixture noise are specified as $\pm\left[\begin{array}{ll}0.033 & 0.52\end{array}\right]$ with corresponding weights of $[0.350 .15]$. In addition, for all cases, the variances of the Gaussian components in the mixture noise are assumed to be the same; that is, $\sigma_{i}=\sigma$ for $i=1, \ldots, M$.

\footnotetext{
${ }^{2}$ This result still holds if the inverses of all the inequality signs are taken.
}

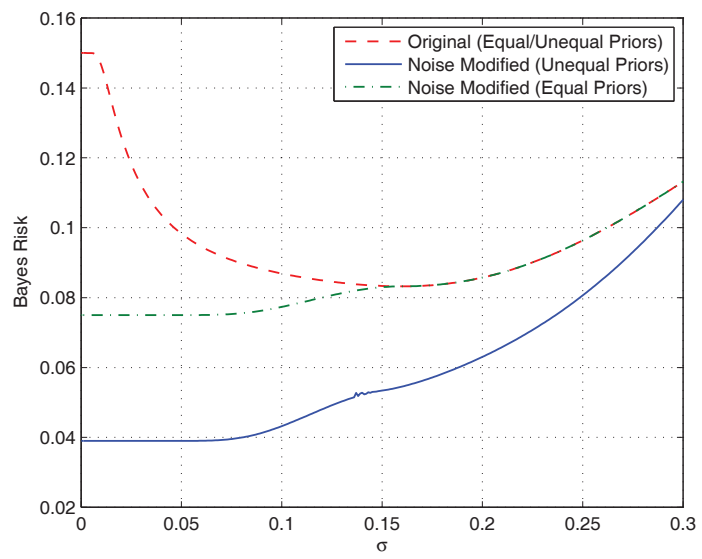

Fig. 1. Bayes risks of the original and noise modified detectors versus $\sigma$ for $\alpha=0.12$ and $A=1$.

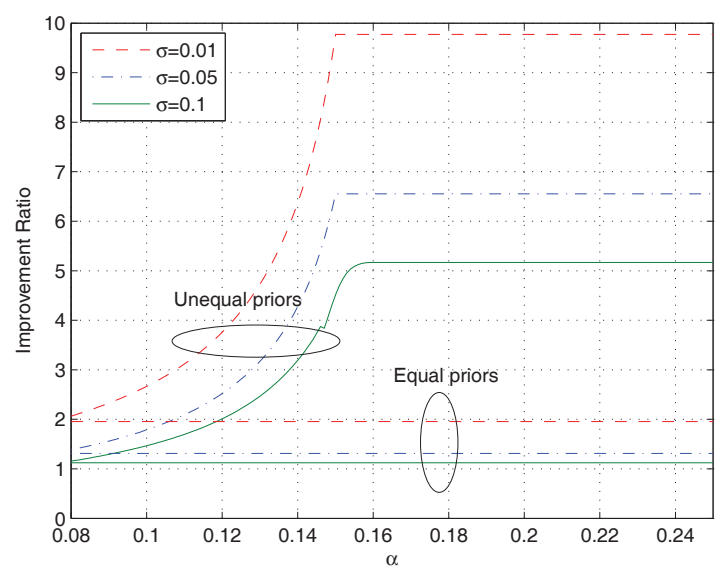

Fig. 2. Improvement ratio versus $\alpha$ for $\sigma=0.01, \sigma=0.05$ and $\sigma=0.1$, where $A=1$.

Fig. 1 illustrates the Bayes risks for the modified and original (i.e., in the absence of additive noise) detectors for various values of $\sigma$ in the cases of equal and unequal priors for $\alpha=0.12$, where $A=1$. It is observed that as $\sigma$ increases, the improvement obtained via additive noise decreases. Also, there is more improvement for the unequal priors case than for the equal priors case, which is expected because there is more room for noise enhancement in the unequal priors case due to the asymmetry between the weights of the conditional risks in determining the Bayes risk.

Fig. 2 illustrates the improvement ratio, defined as the ratio of the Bayes risks without and with additive noise, versus $\alpha$ in the cases of equal and unequal priors for $\sigma=0.01$, $\sigma=0.05$ and $\sigma=0.1$, where $A=1$ is used. In the case of unequal priors, as $\alpha$ increases, an increase is observed in the improvement ratio up to a certain $\alpha$, and then the improvement ratio becomes constant. Those critical $\alpha$ values specify the boundaries between the restricted Bayes and the (unrestricted) Bayes criteria. When $\alpha$ becomes larger than those values, the constraint in (3) is no longer active; hence, the problem reduces to the Bayesian framework. Similarly, as the value of $\alpha$ decreases, the restricted Bayes criterion con- 
Table 1. Optimal additive noise PDFs for various values of $\sigma$.

\begin{tabular}{|c|ccc|}
\hline & \multicolumn{3}{|c|}{$\pi_{0}=0.5 / \pi_{0}=0.9$} \\
\hline$\sigma$ & $\lambda$ & $n_{1}$ & $n_{2}$ \\
\hline \hline 0 & $0.2553 / 0.8$ & $-0.2849 /-0.4063$ & $0.0421 / 0.0598$ \\
\hline 0.08 & $0.4436 / 0.2028$ & $-0.2266 / 0.2266$ & $0.2266 /-0.2266$ \\
\hline 0.15 & $0.7492 / 1$ & $0.0944 /-0.0959$ & $-0.0944 /-$ \\
\hline 0.23 & $1 / 1$ & $0 /-0.0693$ & $-/-$ \\
\hline 0.31 & $1 / 1$ & $0 /-0.0067$ & $-/-$ \\
\hline
\end{tabular}

verges to the minimax criterion. The restricted Bayes criterion achieves its minimum improvement ratio when it becomes equivalent to the minimax criterion and achieves its maximum improvement ratio when it is equal to the Bayes criterion. In the case of equal priors, the improvement ratio is constant with respect to $\alpha$, meaning that the improvement for the minimax criterion equals that for the Bayes criterion. Another observation from the figure is that an increase $\sigma$ reduces the improvement ratio, and for the same values of $\sigma$, there is more improvement for the unequal priors case.

Table 1 shows the optimal additive noise PDFs for various values of $\sigma$ in the cases of equal and unequal priors for $\alpha=0.12$ and $A=1$. According to Proposition 1, the optimal additive noise PDF contains at most two different mass points, which is expressed as $p_{N}(x)=\lambda \delta\left(x-n_{1}\right)+(1-$ $\lambda) \delta\left(x-n_{2}\right)$. From the table, it is observed that the optimal additive noise PDF has two mass points for certain values of $\sigma$ whereas it has a single mass point for other $\sigma$ values. Also, in the case of equal priors, the optimal noise PDFs contain only one mass point at the origin for $\sigma=0.23$ and $\sigma=0.31$, meaning that the detector is nonimprovable in those scenarios. However, there is always improvement for the unequal priors case, which can also be verified from Fig. 1.

Finally, the improvability conditions based on Proposition 3 are evaluated for the considered detection example for various values of $A .^{3}$ The limit on the conditional risks, $\alpha$, is set to the original conditional risks for each value of $\sigma$. For both the equal and unequal priors cases, the improvability conditions state that the detector is improvable for $A=1$ if $\sigma \in[0.005,0.1597]$, for $A=0.9$ if $\sigma \in[0.01,0.1686]$, and for $A=0.8$ if $\sigma \in[0.02,0.161]$. On the other hand, the calculations show that the detector is actually improvable for $A=1$ if $\sigma \leq 0.16$, for $A=0.9$ if $\sigma \leq 0.17$, and for $A=0.8$ if $\sigma \leq 0.161$. The results reveal that the proposed improvability conditions are sufficient but not necessary, and that they are quite effective in determining the range of parameters for which the detector performance can be improved.

In conclusion, the restricted Bayesian framework considered in this study provides a generalization of noise enhanced detection in the minimax and the Bayesian frameworks. In addition, it has practical importance since prior information may not be exact in practice [11].

\section{REFERENCES}

[1] L. Gammaitoni, P. Hanggi, P. Jung, and F. Marchesoni, "Stochastic resonance," Rev. Mod. Phys., vol. 70, no. 1,

${ }^{3}$ The details of the calculations and the related plots are not shown due to space limitations. pp. 223-287, Jan. 1998.

[2] V. Galdi, V. Pierro, and I. M. Pinto, "Evaluation of stochastic-resonance-based detectors of weak harmonic signals in additive white Gaussian noise," Physical Review E, vol. 57, no. 6, pp. 6470-6479, June 1998.

[3] A. Patel and B. Kosko, "Optimal noise benefits in Neyman-Pearson and inequality-constrained signal detection," IEEE Trans. Sig. Processing, vol. 57, no. 5, pp. 1655-1669, May 2009.

[4] P. Hanggi, M. E. Inchiosa, D. Fogliatti, and A. R. Bulsara, "Nonlinear stochastic resonance: The saga of anomalous output-input gain," Physical Review E, vol. 62, no. 5, pp. 6155-6163, Nov. 2000.

[5] S. M. Kay, J. H. Michels, H. Chen, and P. K. Varshney, "Reducing probability of decision error using stochastic resonance," IEEE Sig. Processing Lett., vol. 13, no. 11, pp. 695-698, Nov. 2006.

[6] H. Chen, P. K. Varshney, S. M. Kay, and J. H. Michels, "Theory of the stochastic resonance effect in signal detection: Part I-Fixed detectors," IEEE Trans. Sig. Processing, vol. 55, no. 7, pp. 3172-3184, July 2007.

[7] S. Bayram and S. Gezici, "On the improvability and nonimprovability of detection via additional independent noise," IEEE Sig. Processing Lett., vol. 16, no. 11, pp. 1001-1004, Nov. 2009.

[8] H. Chen, P. K. Varshney, S. M. Kay, and J. H. Michels, "Theory of the stochastic resonance effect in signal detection: Part II-Variable detectors," IEEE Trans. Sig. Processing, vol. 56, no. 10, pp. 5031-5041, Oct. 2007.

[9] S. Bayram and S. Gezici, "Noise-enhanced $M$-ary hypothesis-testing in the minimax framework," in Proc. International Conference on Signal Processing and Commun. Systems, Omaha, Nebraska, Sep. 2009.

[10] E. L. Lehmann, Testing Statistical Hypotheses, Chapman \& Hall, New York, 2 edition, 1986.

[11] Jr. J. L. Hodges and E. L. Lehmann, "The use of previous experience in reaching statistical decisions," The Annals of Mathematical Statistics, vol. 23, no. 3, pp. 396-407, Sep. 1952.

[12] K. E. Parsopoulos and M. N. Vrahatis, Particle swarm optimization method for constrained optimization problems, pp. 214-220, IOS Press, 2002, in Intelligent Technologies-Theory and Applications: New Trends in Intelligent Technologies.

[13] S. M. Kay, "Noise enhanced detection as a special case of randomization," IEEE Sig. Processing Lett., vol. 15, pp. 709-712, 2008.

[14] V. Bhatia and B. Mulgrew, "Non-parametric likelihood based channel estimator for Gaussian mixture noise," Signal Processing, vol. 87, pp. 2569-2586, Nov. 2007. 